\title{
Association between Efficacy of Self-Management to Prevent Recurrences of Depression and Actual Episodes of Recurrence: A Preliminary Study
}

\author{
Mayuko Yamashita \\ Graduate School of Health Sciences, Hiroshima University \\ 1-2-3 Kasumi, Minami-ku, Hiroshima 734-8551, Japan \\ $\&$ \\ School of Human Nursing, University of Shiga Prefecture \\ 2500, Hassaka-cho, Hikone-City, Shiga 522-8533, Japan \\ Tel: 81-749-288-636 E-mail: yamashita.m@nurse.usp.ac.jp \\ Hitoshi Okamura (Corresponding author) \\ Graduate School of Health Sciences, Hiroshima University \\ 1-2-3 Kasumi, Minami-ku, Hiroshima 734-8551, Japan \\ Tel: 81-82-257-5450Ｅ-mail: hokamura@hiroshima-u.ac.jp
}

\author{
Received: August 4, 2011 \\ Accepted: August 31, 2011 Published: December 1, 2011 \\ doi:10.5539/ijps.v3n2p217 \\ URL: http://dx.doi.org/10.5539/ijps.v3n2p217
}

\begin{abstract}
The purpose of this study was to investigate the association between efficacy of self-management to prevent recurrences in patients with depression, and actual episodes of recurrence. We divided 110 patients with depression into a non-recurrence group $(n=60)$ and a recurrence group $(n=50)$, and compared the two groups in regard to socio-demographic and medical variables, scores on the scale for the efficacy of self-management to prevent recurrences of depression, and scores on the Beck's Depression Inventory. The factors associated with episodes of actual recurrence were tested with the logistic regression analysis, and the efficacy of self-management to prevent recurrences of depression was extracted as a factor independently associated with recurrence. The results suggested a statistically significant association between depression recurrence and efficacy of self-management to prevent recurrences of depression. However, the results were inconclusive because of the retrospective, case-control study design.
\end{abstract}

Keywords: Depression, Prevention, Recurrence, Self-efficacy, Self-management

\section{Introduction}

Within a year of the onset of the initial episode of depression 70-80\% of patients who receive treatment are said to experience a remission (Keller et al, 1992; Lam \& Kennedy, 2004). However, as previously reported, the probability of a recurrence and of the probability of a third episode after recurrence are 50-60\% and 70\%, respectively (APA, 2000; Keller \& Roland, 1998). Thus, depression is a disease with a propensity for repeated recurrence. The morbid phase grows longer with the number of recurrences, and the severity of the disease increases. Moreover, after repeated episodes the depression becomes chronic in many patients, and chronic depression may lead to serious social dysfunction, therefore it is important to address the need to prevent depression from recurring (Keller \& Roland, 1998). Previous studies have reported various factors that are associated with recurrences of depression (Angst, 1999; Bruce \& Kim, 1992; Fukuda, Etoh, \& Iwadate, 1983; Harkness, Monroe, Simons, \& Thase, 1999; Lin et al, 1998). Among these factors, the authors paid attention to self-management. Indeed, self-management is considered important to the management of all chronic diseases, including diabetes and renal failure (Kahn \& Weir, 2005), and it involves the control of various aspects of daily living, including disease management and diet modification. In the field of psychiatry the importance of compliance with drug therapy and stress management has been emphasized in the management of schizophrenia 
and depressive disorders. In case of chronic disease such as depression, in particular, it is important to improve the patient's capacity self-management and for physicians to be able to predict whether patients can administer their self-management behavior to prevent recurrences (Finlayson, Edwards, \& Courtney, 2009; Kennedy, Nelson, Reeves, Richardson, Roberts, \& Robinson A, 2004; Robinson, Thompson, Wilkin, \& Roberts, 2001).

One of the criteria for judgment which can predict exactly the administration of self-management behavior is self-efficacy (Bandura, 1977). In the West, some previous reports showed that lower self-efficacy was associated with increased risk of recurrence depression recurrence (Vittengl, Clark, \& Jarrett, 2010; Gopinath, Katon, Russo, \& Ludman, 2007). Therefore, objective indices for the self-efficacy to predict the execution of self-management behavior are required. However, although there were a few self-efficacy scales that assess coping with depression (Perraud, Fogg, Kopytko, \& Gross, 2006), there were no efficacy of self-management scales focused on recurrent episodes of depression. In a previous study, the authors devised a scale to measure the efficacy of self-management to prevent recurrences of depression and evaluated the reliability and validity of the scale (Yamashita \& Okamura, 2008). The present study was designed to determine whether there is an association between efficacy of self-management to prevent recurrences of depression measured by this scale and actual episodes of recurrence.

\section{Methods}

This study was conducted with the approval of the Ethics Committee of the Graduate School of Health Sciences of Hiroshima University and the institutional ethics review board of the participating hospital.

\subsection{Patients}

The study was designed as a retrospective, case-control study. The subjects were 113 outpatients attending the psychiatry clinic of a general hospital in Prefecture F in Japan. They were primarily diagnosed as having depression according to the International Classification of Disease (ICD)-10, and had been followed up since the first episode at the above clinic. The eligibility criteria for subjects were as follows:

\subsubsection{Recurrence Group}

(1) The patient has had 2 or more depression episodes in the period between the first episode and this survey.

(2) The patient is 18 years old or older.

(3) The patient's mental condition is such that the patient does not have any difficulty communicating with others and answering the questionnaire.

(4) The patient has no marked psychiatric symptoms due to complications with other psychiatric disorders.

\subsubsection{Non-recurrence Group}

(1) The patient has had only a single episode, the first one, of depression in the past and no recurrence has been observed for more than 1 year. It has been reported that recurrence of depression occurs most often 6 to 12 months after remission (Kupfer, 1993; Prien \& Kupfer, 1986). Also in Japan, it has been reported in a clinical study of hospitalized patients with depression that most of recurrences occurred within 1 year in the recurrence group (Tadokoro, Miyaoka, \& Kamijima, 2000). Based on these reports, patients without recurrence for more than 1 year were classified as the non-recurrence group in this study.

(2) The patient is 18 years old or older.

(3) The patient's mental condition such that the patient does not have any difficulty communicating with others and answering the questionnaire.

(4) The patient has no marked psychiatric symptoms due to complications with other psychiatric disorders.

\subsection{Measures}

\subsubsection{Socio-demographic and Medical Variables}

Information about the following was collected eliciting answers directly from the patient and by consulting patient's medical records: gender, age, age at the time of diagnosis of the first episode, total number of depressive episodes ever experienced, and interval since the onset at the first episode of depression (interval between diagnosis of the first episode and the initial recurrence in the recurrence group and interval between diagnosis of the first episode and their first examination in the study in the non-recurrence group).

2.2.2 Scale for the Efficacy of Self-management to Prevent Recurrences of Depression (efficacy of self-management scale) (Appendix)

The authors used examples from previous reports and depression guidelines to develop a self-rating (i.e., 
patient's personal rating) scale in Japanese based on factors that have been reported in the literature to increase the risk of depression recurrence, and then evaluated the reliability and validity of the scale (Yamashita \& Okamura, 2008). Cronbach's $\alpha$ was 0.902 , and assessment of construct validity with reference to the correlation between the efficacy of self-management scale and the General Self-efficacy Scale showed a significant correlation (Pearson correlation coefficient $=0.606, \mathrm{p}<0.01$ ). The scale was designed to determine the efficacy of self-management to prevent recurrences of depression and is composed of 4 factors: "life management" (factor 1), "self-control" (factor 2), "self-awareness" (factor 3), and "compliance with treatment" (factor 4). The cumulative contribution ratio of 4 factors was $71.36 \%$. The scale consists of 16 items: 7 items related to factor 1 (0-21 points), which concerns management of daily life to prevent recurrences of depression; 4 items related to factor 2 (0-12 points), which concerns self-control of the patient's emotions and behavior; 3 items related to factor 3 (0-9 points), which concerns self-awareness that is required to prevent recurrences of depression; and 2 items related to factor 4 (0-6 points), which concerns compliance with treatment and its continuation. The scale uses a 4-stage Likert scale to rate for each item according to the patient's level of confidence, thus: "very confident", 3 points, "confident", 2 points, "not very confident", 1 point, and "not confident at all", 0 points. Possible total scores range from 0 to 48 points. High scores indicate greater confidence in efficacy of self-management to prevent recurrences of depression.

\subsubsection{Self-rating Depression Scale (Beck's Depression Inventory, BDI)}

This BDI is a self-rating scale based on patients' verbatim descriptions to assess the severity of depression during the previous 1-week period that was devised by Beck, Ward, Mendelson, Mock, and Erbaugh (1961) based on clinical observations and patients' complaints. It is composed of 21 items that include "sorrow" and "sense of self-reproach". Patients select the sentence that corresponds best to their condition and possible total scores range from 0 to 63 points. Higher scores indicate more severe depression. Depression is classified into three stages of severity based on the total score on the BDI: no depression (0-13 points); mild to moderate depression (14-24 points); and severe depression (25 points or over) (Beck, 1967). The Japanese version of the scale was developed by Hayashi (1988). The split-half reliability coefficient was 0.62 , and Pearson correlation coefficient between the BDI score and scores of depressive tendency in Yatabe-Guilford personality inventory was 0.62.

\subsection{Survey Methods}

All of the patients who attended the outpatient clinic already mentioned between April and June of 2008 were included in the target population of this study. Before the patients who met the eligibility criteria were interviewed, the attending physicians explained the purpose of the study to them. After the study was explained to the patient again by the first author, patients who consented to participate in this study and signed the consent form were interviewed at this study entry, at which point the efficacy of self-management scale and the BDI scale were administered.

\subsection{Statistical Analysis}

(1) The demographic characteristics (age, gender, age at the time of diagnosis of the first episode, interval after the initial episode) and mental condition (BDI score) of the non-recurrence group and recurrence group were compared with the $t$-test or chi-square test after confirming the normality of the data.

(2) The total score and the score for each item on the efficacy of self-management scale were calculated in each group to assess the possibility of discriminating between the non-recurrence group and the recurrence group on the basis of efficacy of self-management to prevent recurrences of depression. The $t$-test was used to analyze the data in the two groups for significant differences.

(3) Factors that might be related to increased risk of depression recurrence were assessed with a logistic regression analysis (forced input method) by using the presence/absence of recurrences as the dependent variable and the variables identified by the univariate analysis as significantly different between the two groups as the independent variables. Furthermore, discriminant analysis was conducted as it can highlight predictors related to the presence/absence of recurrences.

(4) To assess any association between efficacy of self-management to prevent recurrences of depression and the number of recurrences in the past, the patients in the recurrence group were divided further into a subgroup with a history of only one recurrence (recurrence group A) and a subgroup with a history of two or more recurrences (recurrence group B). The mean total score and mean scores for each item on the efficacy of self-management scale were calculated in each subgroup and the data were analyzed for significant differences between them by the $t$-test.

(5) In order to test the specificity and sensitivity of the efficacy of self-management scale, receiver operating 
characteristics (ROC) analysis was performed. Representing ROC analysis on a curve is a way of expressing the relationship between the true positive rate (sensitivity) and the false-positive rate ( 1 - specificity). The curve is a representation of the ability of the screening instrument to discriminate between "cases" and "non-cases". The desired cut-off point is generally chosen in order to minimize the sum of false-positive and false-negative test results.

The $\mathrm{P}$ values in all of the tests were two-sided, and $\mathrm{p}$ values $<0.05$ were considered significant. Statistical Package for the Social Sciences (SPSS) Statistics ver. 17.0 for Windows software was used to carry out all of the statistical analyses.

\section{Results}

\subsection{Subjects' Characteristics (Table 1)}

Of the total of 113, the 110 who met the eligibility criteria for the study and from whom informed consent was obtained were included in the analysis. Sixty subjects, 24 males (40.0\%) and 36 females $(60.0 \%)$, with a history of only one episode of depression were assigned to the non-recurrence group, and the other 50 subjects, 29 males $(58.0 \%)$ and 21 females $(42.0 \%)$, who had a past history of at least one recurrence were assigned to the recurrence group. Comparison of the characteristics of the two groups revealed significant differences only in the subject's age at the time of diagnosis of the first episode of depression.

3.2 Assessment of the Possibility of Discriminating between the Non-recurrence Group and the Recurrence Group on the Basis of Efficacy of Self-management to Prevent Recurrences of Depression

To assess the possibility of discriminating between the non-recurrence group and the recurrence group in terms of efficacy of self-management to prevent recurrences of depression, the mean total score and the mean score for each factor on the efficacy of self-management scale were calculated in each of the two groups. Analysis with the $t$-test revealed that the mean total score and the score for factor 1, factor 2, and factor 3 were significantly higher in the non-recurrence group than in the recurrence group. Although the difference between the two groups in the score for factor 4 was not significant, the score for factor 4 tended to be slightly higher in the non-recurrence group (Table 2).

\subsection{Assessment of Factors Related to Depression Recurrence}

Factors related to depression recurrence were assessed with a logistic regression analysis (forced input method). The presence/absence of recurrences of depression was used as the dependent variable, and the age at the time of diagnosis of the first episode and total score on the efficacy of self-management scale, both of which had been identified by the univariate analysis as significantly different between the non-recurrence group and recurrence group, and the BDI score were used as the independent variables. The results revealed that the three variables were independently related to depression recurrence (Table 3). Furthermore, discriminant analysis (Table 4) yielded a statistically significant function explaining 53.5\% (Wilks' lambda, 0.72; df, 6; p < 0.001). In particular, total score on the efficacy of self-management scale was shown to play an important role in the discrimination.

\subsection{Assessment of Efficacy of Self-management to Prevent Recurrences of Depression in Relation to the Number of Recurrences of Depression}

To assess any association between efficacy of self-management to prevent recurrences of depression and the number of recurrences, the patients in the recurrence group were divided into recurrence group A (one instance of recurrence) and recurrence group B (two or more episodes). The mean total score and the mean score for each item on the scale were calculated in each subgroup, and the data for the significance of differences between the two groups were analyzed with the $t$-test. The results showed no significant differences between the two subgroups in the total score or the scores for any of the items (Table 5).

3.5 Screening for Discriminating between the Non-recurrence Group and the Recurrence Group by the Efficacy of Self-management Scale

From the ROC curve, the cut-off point for the screening seemed to $22 / 23$. This cut-off point is associated with $76.0 \%$ sensitivity and $76.7 \%$ specificity (positive predictive value [PPV]: $78.3 \%$, negative predictive value [NPV]: 78.1\%).

\section{Discussion}

To assess the association between efficacy of self-management to prevent recurrences of depression and actual episodes of recurrence, we first investigated the possibility of using scores on the efficacy of self-management scale to discriminate between the non-recurrence group and the recurrence group. The results showed that the total score and scores for factors 1,2 , and 3 were significantly higher in the non-recurrence group, suggesting the 
possibility of using the scale to discriminate between the non-recurrence group and the recurrence group. The scale included the following items to assess the efficacy of self-management, which is considered necessary to prevent recurrences of depression, and the self-efficacy of self-awareness, which is considered necessary for self-management: compliance with treatment, coping with stress, self-knowledge, and actual use of social support. Scores were assigned on the basis of the patient's level of confidence, that is, higher scores were assigned for higher levels of confidence. Recognition of the need for self-efficacy results in improved performance of the activities, and the efficacy of self-management has a long-term influence on an individual's future behavior (Bandura, 1977). Therefore, it is suggested that clear and strong recognition of the need for efficacy of self-management to prevent recurrences of depression allows a positive approach to problems and taking steps to perform the appropriate self-management behavior that is needed to prevent recurrences of depression. In the present study, the scores on the efficacy of self-management scale were significantly higher in the non-recurrence group, which allowed discrimination between the non-recurrence group and the recurrence group, showing that patients who have a high degree of awareness of efficacy of self-management may have the ability to behave appropriately to prevent recurrences. The difference in the score on the factor 4 items, on the other hand, was not significant. Factor 4 items include "the patient can continue attending a hospital" (Lewis, Marcus, Olfson, Druss, \& Pincus, 2004) and "the patient can continue taking medicine as indicated by the physician" (Angst, 1999). The subjects of this study were all outpatients, and since their mental condition was relatively stable, their compliance with treatment is assumed to have been favorable. Because patients whose compliance with treatment was poor, who stopped attending the outpatient clinic, and who no longer required drug therapy or outpatient care were excluded from the study, there was bias in the distribution of the scores for the factor 4 items. The bias on the selection of the subjects may have affected the results.

The association between efficacy of self-management to prevent recurrences of depression and actual episodes of recurrence was tested with the logistic regression analysis, and the efficacy of self-management was extracted as a factor independently associated with recurrence, as well as age at the time of diagnosis of the first episode and the BDI score. Some studies have pointed out that a younger age at the time of diagnosis of the first episode increases the risk for depression recurrence (Fukuda, Etoh, \& Iwadate, 1983; Hirschfeld RM, 2001), and the present finding is in keeping with that of previous reports.

Regarding the efficacy of self-management to prevent recurrences of depression, which were associated with actual episodes of recurrence independently of the severity of depression, factor 1 (life management) in the questionnaire is composed of items to assess self-efficacy in regard to matters related to self-management, including indirect behavior as well as direct behavior to prevent recurrences of depression. The low self-efficacy in factor 1 is suggested to cause chronic fatigue and to make it difficult to improve human relations in areas outside of work or to engage in hobbies and amusements in daily living (Gunther, Roick, Angermeyer, \& Konig, 2008; Tellenbach, 1961), which results in mental and/or physical instability and a higher risk of depression recurrence. Factor 2 (self-control) is composed of items to assess self-efficacy in regard to matters related to control of the patient's emotions and behavior. Depressive patients often exhibit three characteristic cognitive patterns: negative estimation of themselves, negative interpretation of experiences, and negative views of the future (Beck, 1983; Hyde, Mezulis, \& Abramson, 2008). Because such cognitive patterns are important elements in inducing exacerbations and recurrences of depression, it is suggested that failure of emotional control results in various negative experiences, and increases the risk of depression recurrence (Blackburn \& Moore, 1997). Factor 3 (self-awareness) is composed of items related to self-efficacy in regard to matters related to self-awareness. When there is inadequate self-knowledge regarding stresses to which the patient is vulnerable, the cause of the patient's depression, and situations that increase the risk of recurrences and exacerbation of depression, the living and working situation becomes similar to the situation after depression recurrence and probably increases the risk of depression recurrence.

Self-efficacy can be cultivated, i.e., it can be improved. Some studies have shown that it can be improved by adopting approaches to desirable health actions. On the other hand, self-efficacy can be impaired by negative experiences, such as failure. When this is taken into consideration, there is the possibility that repeated recurrence decreases the efficacy of self-management to prevent recurrences of depression. To verify this possibility, patients in the recurrence group were divided into a group with a history of one recurrence and a group with a history of at least two recurrences, and the scores of the two subgroups on the efficacy of self-management scale were compared. The results showed no significant difference in either the total or mean scores for items on the scale, providing evidence against the notion that efficacy of self-management decreases with the number of recurrences and suggesting that the self-efficacy of the patients in the recurrence group was lower regardless of the number of recurrences. The mean interval between the first episode and the initial 
recurrence was 4.4 years (SD: 4.5 years) in recurrence group A and 5.3 years (SD: 3.1 years) in recurrence group B. The mean interval in the recurrence group was 4.8 years (SD: 4.0 years). The mean interval between the date of diagnosis of the first episode and the date of the first examination in this study was longer, 6.12 years (SD: 7.1 years), in the non-recurrence group, indicating that the risk of recurrence is not necessarily related to the interval since the onset at the first episode.

A major limitation of the present study is that this was a retrospective, case-control study. Therefore, it was impossible to clearly demonstrate a causal relationship between the self-efficacy and recurrences. Second, this was a small-scale study and patients were recruited from only one clinic. Although the clinic was a general psychiatric clinic in Japan, this made it difficult to generalize the results. To determine whether there is a causal relationship between self-efficacy and recurrences, cohort studies or collecting data on both variables in randomized controlled depression treatment trials with appropriate follow-up lengths to capture early recurrences of depression should be conducted. Third, the subjects in this study were surveyed between April and June. Therefore, issues of seasonality on recurrence cannot be ruled out.

\section{Acknowledgments}

The authors are grateful to Dr. Shigeki Yamamura, Department of Psychiatry, Obama Municipal Hospital, for their advice and research assistance.

\section{Conflict of Interest}

The authors have no conflict of interest with any commercial or other associations in connection with the submitted article.

\section{References}

American Psychiatric Association. (2000). Diagnostic and statistical manual of mental disorders, 4th ed., text revision. Washington DC: APA.

Angst, J. (1999). Major depression in 1998: are we providing optimal therapy? Journal of Clinical Psychiatry, $60,5-9$

Bandura, A. (1977). Self-efficacy: toward a unifying theory of behavior change. Psychological Review, 84, 195-215. http://dx.doi.org/10.1037/0033-295X.84.2.191

Beck, A. T. (1967). Depression; clinical, experimental, and theoretical aspects. London: Harpere and Row.

Beck, A. T. (2008). The evolution of the cognitive model of depression and its neurobiological correlates. American Journal of Psychiatry, 165, 969-977. http://dx.doi.org/10.1176/appi.ajp.2008.08050721

Beck, A.T., Ward, C. H., Mendelson, M., Mock, J., \& Erbaugh, J. (1961). An inventory for measuring depression. Archives of General Psychiatry, 4, 561-571

Blackburn, I. M., \& Moore, R. G. (1997). Controlled acute and follow-up trial of cognitive therapy and pharmacotherapy in out-patients with recurrent depression. British Journal of Psychiatry, 171, 328-334. http://dx.doi.org/10.1192/bjp.171.4.328

Bruce, M. L., \& Kim, K. M. (1992). Differences in the effects of divorce on major depression in men and women. American Journal of Psychiatry, 149, 914-917

Finlayson, K., Edwards, H., \& Courtney, M. (2009). Factors associated with recurrence of venous leg ulcers: a survey and retrospective chart review. International Journal of Nursing Studies, 46, 1071-1078, http://dx.doi.org/10.1016/j.ijnurstu.2008.12.012

Fukuda, K., Etoh, T., \& Iwadate, T. (1983). The course and prognosis of manic- depressive psychosis: a quantitative analysis of episodes and intervals. Tohoku Journal of Experimental Medicine, 139, 299-307. http://dx.doi.org/10.1620/tjem.139.299

Gopinath, S., Katon, W. J., Russo, J. E., \& Ludman, E. J. (2007). Clinical factors associated with relapse in primary care patients with chronic or recurrent depression. Journal of Affective Disorders, 101, 57-63. http://dx.doi.org/10.1016/j.jad.2006.10.023

Gunther, O. H., Roick, C., Angermeyer, M. C., \& Konig, H. H. (2008). The responsiveness of EQ-5D utility scores in patients with depression: A comparison with instruments measuring quality of life, psychopathology and social functioning. Journal of Affective Disorders, 105, 81-91. http://dx.doi.org/10.1016/j.jad.2007.04.018

Harkness, K. L., Monroe, S. M., Simons, A. D., \& Thase, M. (1999). The generation of life events in recurrent $\begin{array}{llll}\text { and non-recurrent } & \text { depression. } & \text { Psychological } & \text { Medicine, }\end{array}$ 
http://dx.doi.org/10.1017/S0033291798007752

Hayashi, K. (1988). Study on depressive states of students based on Beck's cognitive therapy (Japanese). Student Counseling Journal, 9, 97-107

Hirschfeld, R.M. (2001). Clinical importance of long-term antidepressant treatment. British Journal of Psychiatry, 42 (Suppl), S4-8. http://dx.doi.org/10.1192/bjp.179.42.s4

Hyde, J. S., Mezulis, A. H., \& Abramson, L. Y. (2008). The ABCs of depression: integrating affective, biological, and cognitive models to explain the emergence of the gender difference in depression. Psychological Review, 115, 291-313. http://dx.doi.org/10.1037/0033-295X.115.2.291

Kahn, C. R., \& Weir, G.C. (2005). Joslin's Mellitus. Philadelphia: Joslin Diabetes Center.

Keller, M. B., \& Boland, R.J. (1998). Implications of failing to achieve successful long-term maintenance treatment of recurrent unipolar major depression. Biological Psychiatry, 44, 348-360. http://dx.doi.org/10.1016/S0006-3223(98)00110-3

Keller, M. B., Lavori, P. W., Mueller, T. I., Endicott, J., Coryell, W., \& Hirschfeld, R. M., et al. (1992). Time to recovery chronicity and levels of psychopathology in major depression. A 5-year prospective follow-up of 431 subjects, Archives of General Psychiatry, 49, 809-816

Kennedy, A. P., Nelson, E., Reeves, D., Richardson, G., Roberts, C., Robinson, A., et al. (2004). A randomised controlled trial to assess the effectiveness and cost of a patient orientated self management approach to chronic inflammatory bowel disease. Gut, 53, 1639-1645. http://dx.doi.org/10.1136/gut.2003.034256

Kupfer, D. J. (1993). Management of recurrent depression. Journal of Clinical Psychiatry, 54 (suppl 2), 29-33

Lam, R. W., \& Kennedy, S.H. (2004). Evidence-based strategies for achieving and sustaining full remission in depression: focus on metaanalyses. Canadian Journal of Psychiatry, 49, 17S-26S. [Online] Available: http://www.cpa-apc.org/index.php

Lewis, E., Marcus, S. C., Olfson, M., Druss, B. G., \& Pincus, H. A. (2004). Patient's early discontinuation of antidepressant prescriptions. Psychiatric Services, 55, 494. http://dx.doi.org/10.1176/appi.ps.55.5.494

Lin, E. H., Katon, W. J., Vonkorff, M., Russo, J. E., Simon, G. E., \& Bush, T. M., et al. (1998). Relapse of depression in primary care: rate and clinical predictors. Archives of Family Medicine, 7, 443-449. http://dx.doi.org/10.1001/archfami.7.5.443

Perraud, S., Fogg, L., Kopytko, E., \& Gross, D. (2006). Predictive validity of the Depression Coping Self-Efficacy Scale (DCSES). Research in Nursing \& Health, 29, 147-160. http://dx.doi.org/10.1002/nur.20126

Prien, R. F., \& Kupfer, D. J. (1986). Continuation drug therapy for major depressive episodes: how long should it be maintained? American Journal of Psychiatry, 143, 18-23

Robinson, A., Thompson, D. G., Wilkin, D., \& Roberts, C. (2001). Northwest Gastrointestinal Research Group. Guided self-management and patient-directed follow-up of ulcerative colitis: a randomised trial. Lancet, 358, 976-981. http://dx.doi.org/10.1016/S0140-6736(01)06105-0

Tadokoro, C., Miyaoka, H., \& Kamijima, K. (2000). Risk factors for relapse or recurrence in patients with depression (Japanese). Seishin Igaku, 42, 591-597

Tellenbach, H. (1961). Melancholie. Berlin: Springer-Verlag.

Vittengl, J. R., Clark, L. A., \& Jarrett, R. B. (2010). Moderators of continuation phase cognitive therapy's effects on relapse, recurrence, remission, and recovery from depression. Behaviour Research and Therapy, 48, 449-458. http://dx.doi.org/10.1016/j.brat.2010.01.006

Yamashita, M., \& Okamura, H. (2008). Development of a self-efficacy scale to measure self-management behavior related to the prevention of recurrence of depression and assessment of its reliability and validity (Japanese). Japanese Journal of Clinical Psychiatry, 37, 1045-1052 
Table 1. Comparison between the characteristics of the subjects in the non-recurrence group and recurrence group

\begin{tabular}{|c|c|c|c|}
\hline & $\begin{array}{l}\text { Non-recurrence group } \\
(\mathrm{N}=60)\end{array}$ & $\begin{array}{l}\text { Recurrence group } \\
(\mathrm{N}=50)\end{array}$ & $\mathrm{P}^{\mathrm{a}}$ \\
\hline Age (years) & $\begin{array}{l}55.1(\text { SD 20.0) } \\
\text { (range 22-82) }\end{array}$ & $\begin{array}{l}54.4(\mathrm{SD} 18.0) \\
\text { (range 21-88) }\end{array}$ & 0.850 \\
\hline $\begin{array}{l}\text { Gender } \\
\quad \text { Male } \\
\text { Female }\end{array}$ & $\begin{array}{l}24(40.0 \%) \\
36(60.0 \%)\end{array}$ & $\begin{array}{l}29(58.0 \%) \\
21(42.0 \%)\end{array}$ & 0.060 \\
\hline $\mathrm{BDI}^{\mathrm{b}}$ score & $\begin{array}{l}15.3(\text { SD 11.9) } \\
\text { (range 2-37) }\end{array}$ & $\begin{array}{l}19.0(\text { SD 10.2) } \\
\text { (range 2-35) }\end{array}$ & 0.092 \\
\hline $\begin{array}{l}\text { Age at the time of diagnosis } \\
\text { of the first episode (years) }\end{array}$ & $\begin{array}{l}49.0(\text { SD 20.3) } \\
\text { (range 13-78) }\end{array}$ & $\begin{array}{l}38.6(\text { SD 16.7) } \\
\text { (range 12-75) }\end{array}$ & 0.004 \\
\hline $\begin{array}{l}\text { Interval after the initial } \\
\text { episode (years) }\end{array}$ & $\begin{array}{l}6.1(\text { SD } 7.1) \\
\text { (range 1-27) }\end{array}$ & $\begin{array}{l}4.8(\text { SD } 4.0) \\
\text { (range } 0-18)\end{array}$ & 0.239 \\
\hline
\end{tabular}

a: $t$-test or chi-square test, b: Beck's Depression Inventory

c: Interval between diagnosis of the first episode and their first examination in the study in the non-recurrence group.

Interval between diagnosis of the first episode and the initial recurrence in the recurrence group

Table 2. Comparison between the scores of the non-recurrence group and the recurrence group on the efficacy of self-management scale

\begin{tabular}{|l|c|c|c|}
\hline & $\begin{array}{c}\text { Scores of the } \\
\text { non-recurrence group } \\
(\mathrm{N}=60)\end{array}$ & $\begin{array}{c}\text { Scores of the } \\
\text { recurrence group } \\
(\mathrm{N}=50)\end{array}$ & $\mathrm{P}^{\mathrm{a}}$ \\
\hline Total & 28.4 (SD 8.4) & 19.9 (SD 6.7) & $<0.001$ \\
Factor 1 & 12.1 (SD 4.5) & 7.8 (SD 3.7) & $<0.001$ \\
Factor 2 & 6.1 (SD 3.1) & 3.6 (SD 2.4) & $<0.001$ \\
Factor 3 & 5.4 (SD 2.2) & 4.2 (SD 2.3) & 0.004 \\
Factor 4 & 4.7 (SD 1.3) & 4.3 (SD 1.2) & 0.095 \\
\hline
\end{tabular}

a: $t$-test

Factor 1: life management, Factor 2: self-control, Factor 3: self-awareness, Factor 4: compliance with treatment

Table 3. Factors related to depression recurrence - logistic regression analysis

\begin{tabular}{|l|c|c|c|c|c|}
\hline & $\begin{array}{c}\text { Estimate } \\
\text { (beta) }\end{array}$ & $\begin{array}{c}\text { Standard } \\
\text { error }\end{array}$ & Odds ratio & $\begin{array}{c}95 \% \\
\text { confidence } \\
\text { interval }\end{array}$ & $\mathrm{P}$ \\
\hline $\begin{array}{l}\text { Total score on the efficacy of } \\
\text { self-management scale }\end{array}$ & -0.180 & 0.042 & 0.836 & $0.770-0.907$ & $<0.001$ \\
\hline BDI score & -0.054 & 0.027 & 0.947 & $0.898-1.000$ & 0.048 \\
\hline $\begin{array}{l}\text { Age at the time of diagnosis of the } \\
\text { first episode }\end{array}$ & -0.025 & 0.013 & 0.976 & $0.952-1.000$ & 0.048 \\
\hline
\end{tabular}


Table 4. Summary of discriminant analysis

\begin{tabular}{|l|c|}
\hline Predictor & $\begin{array}{c}\text { Standardized } \\
\text { canonical discriminant function coefficient }\end{array}$ \\
\hline Total score on the efficacy of self-management scale & 1.071 \\
BDI score & 0.426 \\
Age at the time of diagnosis of the first episode & 0.386 \\
\hline Canonical correlation & 0.535 \\
Eigen value & 0.402 \\
Wilks' lambda & 0.713 \\
Chi square & $35.989 ; \mathrm{df}=3$ \\
\hline
\end{tabular}

df: degrees of freedom

Table 5. Comparison between the scores of the recurrence group A and the recurrence group B on the efficacy of scale self-management scale

\begin{tabular}{|l|c|c|c|}
\hline & $\begin{array}{c}\text { Scores of the } \\
\text { recurrence group A } \\
(\mathrm{N}=28)\end{array}$ & $\begin{array}{c}\text { Scores of the } \\
\text { recurrence group B } \\
(\mathrm{N}=22)\end{array}$ & $\mathrm{P}^{\mathrm{a}}$ \\
\hline Total & 19.2 (SD 5.8) & 20.8 (SD 7.7) & 0.410 \\
Factor 1 & $7.4($ SD 3.4) & 8.2 (SD 4.1) & 0.476 \\
Factor 2 & 3.5 (SD 2.1) & 3.7 (SD 2.8) & 0.782 \\
Factor 3 & 3.7 (SD 2.0) & 4.8 (SD 2.5) & 0.093 \\
Factor 4 & 4.5 (SD 1.1) & 4.0 (SD 1.3) & 0.236 \\
\hline
\end{tabular}

a: $t$-test

Recurrence group A: a subgroup with a history of only one recurrence

Recurrence group B: a subgroup with a history of two or more recurrences

Factor 1: life management, Factor 2: self-control, Factor 3: self-awareness, Factor 4: compliance with treatment 
Appendix. Items of the scale for the efficacy of self-management to prevent recurrences of depression
a. You can continue to attend the hospital.
b. You can continue to take your medication as instructed by physicians even if it is over a long-term period.
c. You can ask the physicians and nurses what you do not understand and what you want to know.
d. You can take sufficient rest when tired.
e. When there are any changes in your current symptoms and conditions, you can report them to the physicians by yourself.
f. When you are suspicious of or dissatisfied with the method of treatment, you can tell the physicians and nurses what your problems are.
g. You can actively participate in the treatment.
h. You can get enough hours of sleep every day.
i. When you have any difficulties or worries, you can consult someone about them.
j. When necessary, you can receive support using social resources such as public health and welfare.
k. You can incorporate play and humor into your life.
1. When you feel pessimistic about things or feel like blaming yourself, you can stop and correct the thought.
$\mathrm{m}$. You can ask someone for help when you need it, without trying to carry everything on your own shoulders.
$\mathrm{n}$. When you have severe anxiety or stress, you can relax in your own way.
o. When you feel depressed or anxious, you can try to change your mood in a positive way.
p. You can enjoy your free time with hobbies and other pastimes.
q. You should always look at yourself objectively.
r. You can predict situations that cause recurrence and worsening of depression, and avoid them.
s. You can prevent recurrence and worsening of your depression.
t. You can tell yourself what you cannot do without pushing yourself too hard.
u. You can understand the reason why you developed depression.
v. You can reserve energy for everything without pushing yourself too hard.
w. You can understand what gives you severe stress, and try to avoid the cause.
$\mathrm{x}$. You can be kind to yourself.
y. You can change your way of thinking from negative to positive.
z. You can accept your disease.
aa. You can manage your health.
ab. You can trust your current physician.
ac. You can take care of your body.
ad. You can believe that the depression will surely get better. 